\title{
Modeling of Vascular Systems
}

Ph.D. theses

\section{Róbert Kákonyi}

Supervisors:

Dr. Miklós Erdélyi

Dr. Gábor Szabó

Doctoral School in Physics

Department of Optics and Quantum Electronics

University of Szeged

Faculty of Science and Informatics

Szeged

2010 


\section{Introduction}

The technical background of x-ray generation equipments has significantly improved in the last 115 years since the discovery of x-rays. However, the phenomenon used to generate $\mathrm{x}$-rays in the state of the art medical x-ray tubes is the same phenomenon examined during the discovery of the x-rays. That is, an impacting electron beam into the surface of a metallic electrode in vacuum results in emerging $x$-rays.

The direction of the technical development points into the direction decreasing focal spot size, increasing thermal performance and increasing maximum tube current. As anode material in modern x-ray tubes tungsten and its alloys are used, while in the case of mammographic tubes molybdenum and rhenium are used instead of the initially used platinum. A significant increase in the maximum tube current was made possible through the introduction of rotating anode which distributes the anode heat to a larger surface. Increasingly accurate modeling is required for further development of x-ray units.

The physical phenomena to be investigated during imaging could be classified into one of the three general structural categories: the source, the object under investigation and the detector. In all three categories, the phenomena strongly depend on the properties of x-ray used for imaging, namely the photon energy or spectrum. Depending on the application, this energy dependence can be disadvantageous, for example the beam hardening in the case of CT-s, or beneficial when this energy dependence is used to get more information about the object, such as in the case of dual energy material decomposition. In both cases, the source spectrum has a significant impact on the estimation of the object parameters, and its knowledge on the accuracy of the estimation.

In my dissertation I reviewed the current models of x-ray tubes' spectra used in the field of medical imaging. The attempts to model the x-ray spectra emergent from the anode of the x-ray tube can be classified into several categories based on the used methods: 1. empirical, 2. semi-empirical, 3. Monte Carlo.

In the case of empirical and semi-empirical methods, the parameters of a mathematical formula based on the properties of physical phenomena are fit on measured data. The predictive capabilities of the model are limited by the approximations and the phenomena taken into consideration during the derivation of the used formula. Despite these constraints, the parameters of the $\mathrm{x}$-ray spectra emergent from the anode can be well estimated by means of empirical and semi-empirical methods. 
The expansion of computing capacity made it possible to have high resolution Monte Carlo results about the spectra of the x-ray radiation emergent from the anode. The Monte Carlo method has the advantage that all the phenomena of the transport of photons and electrons and their coupling (e.g. an electron excites a photon or an electron, then the excited particle excites another electron or photon, and so on) can be easily taken into account with high accuracy. The drawback is its statistical nature, i.e. if the uncertainty of the simulation results shall be reduced by half or the energy resolution of the calculated spectra shall be doubled the simulation run time is quadrupled. This limit is significantly reduced by the computation capacities available nowadays.

Prior investigations carried out by means of empirical models have shown that the structure of anode surface has a significant impact on the properties of the x-ray radiation from practical point of view. Certain patents suggest appropriate modifications of the anode surface to improve thermal properties.

\section{Objectives}

The present thesis arose from cooperation between GE Healthcare and the University of Szeged, Department of Optics and Quantum Electronics. One of the aims of our cooperation is to improve the physical models of XTOP (X-ray Technique Optimization Platform) simulation tool. During the engineering work optimization of the control system of Innova X-ray vascular imaging systems is carried out by means of the XTOP simulation tool, so accuracy is in explicit connection with the time spent for checking the design and fabrication and the used human resources.

My overall goal is to improve the computation accuracy of the imaging parameters and to develop the appropriate calibration techniques. For the sake of reasonability and because of the schedule of the project, first I will examine the model of the x-ray source and its development possibilities. Currently the IPEM (Institute of Physics and Engineering in Medicine) $\mathrm{x}$-ray spectrum table is used in XTOP to estimate the x-ray spectra in the direction of the anode angle. The IPEM spectrum table is based on the BM (Birch and Marshall) model, fitting the model's parameters on measurement data. The IPEM table covers the 30 to $150 \mathrm{kVp}$ tube voltage range with $1 \mathrm{kVp}$ resolution and approximately the $6-22^{\circ}$ emission angle range with $1^{\circ}$ increments. Since the flat panel detector in the Innova systems covers approximately the $2-22^{\circ}$ emission angle range, the IPEM table is insufficient to define a 
photon source which covers the necessary emission angle range in further simulations. Furthermore it is obvious from the derivation of the BM model that the anisotropy of the bremsstrahlung is neglected. For these reasons, I will create an entirely new spectrum table for further simulations.

It is known from the literature that the surface roughness has an impact on the properties of the spectra of the x-ray radiation emerging from the focal spot and the aging properties of x-ray tubes from different manufacturers may differ. Therefore I will examine the properties of the anode surface roughness of Maxiray 150 type x-ray tubes which are used in Innova systems.

I will perform Monte Carlo simulations to analyze the effect of anode surface roughness from the examination results of the Maxiray 150 tubes and using anode profiles adopted from the literature. My final goal is create spectrum tables for the used profiles, which will be used for simulation of the whole system.

\section{Measurement and simulation methods}

The anode surface of Maxiray 150 type x-ray tubes used in Innova systems were examined by means of optical microscope, profilometer, and scanning electron microscope. The results of these studies and profiles adopted from the literature were used in further simulations.

The simulations were performed by means of the MCNPX (Monte Carlo N Particle eXtended) Monte Carlo particle transport code which is a very common and frequently used tool in simulation of medical imaging equipments. Simulation of the photon flux was investigated in different geometric layouts. Point detectors were used to calculate photon flux.

Two different simulation geometries were used during the simulations. The angular and energy distribution of the photon flux emerging from the focal spot was determined by means of the first geometry. The second geometry was a simple model of the Innova 3100 vascular imaging system. 


\section{Results}

I have analyzed the properties of the x-ray beam emerging from tungsten anode X-ray tubes by Monte Carlo simulation. I summarize my results in the following points:

1. A simple model was developed to describe the effect of anode surface roughness, based on the experimental analysis of 19 Maxiray 150 type x-ray tubes. The surfaces consisted of $\sim 100 \mu \mathrm{m}$ sized islands separated by cracks of $8 \mu \mathrm{m}$ average depth. The typical width of these cracks was a few $\mu \mathrm{m}$-s, but they might reach $20 \mu \mathrm{m}$. The surface roughness of the islands' surface did not exceed $1 \mu \mathrm{m}$. Using these results a one dimensional surface model was developed, $8 \mu \mathrm{m}$ deep cracks were inserted into an ideal plane surface with $150 \mu \mathrm{m}$ period. The width of the cracks was chosen corresponding to the anode surface roughness.

2. I have analyzed the x-ray flux emergent from an ideal plane anode surface by Monte Carlo simulation with high energy and angular resolution $\left(0.5 \mathrm{keV}\right.$ and $1^{\circ}$ respectively). In spite of the current knowledge in the literature I concluded that some of the spectral components have definite maxima as a function of emission angle. The position of the maxima strongly depends on the photon energy, but not in the form of any analytical function. I have experimentally verified the existence of the phenomenon.

3. I have analyzed the x-ray flux emergent from anode surfaces with different profiles by means of Monte Carlo simulation with high energy and angular resolution $(0.5 \mathrm{keV}$ and $1^{\circ}$ respectively). In the literature the effect of anode surface roughness was attempted to be described by a filter made of the anode material. I have shown that this simplification leads to strongly parameter dependent error by comparing to the newly examined effect of anode surface roughness.

4. I ascertained that the effect of anode surface roughness has high impact on the emission angle dependence of the spectral photon flux. The position of maxima of the spectral photon flux shifts to higher emission angles by increasing the anode surface roughness. Angular position of maxima of the spectral photon flux is $90^{\circ}$ above approximately $55 \mu \mathrm{m}$ anode surface roughness value in all energy ranges, thus the spectral photon flux monotonically increases with emission angle. 
5. I have analyzed the physical quantities which determine the important beam properties as a function of emission angle and anode surface roughness. I have concluded that anode surface roughness could be used to change the beam properties appropriately. Such property is the emission angle dependence of the beam hardness. Increasing the anode surface roughness decreases the emission angle dependence of the HVL (half value layer), which describes the beam hardness, while increases the emission angle dependence of the intensity. Furthermore I concluded that there exists an emission angle range where the HVL value does not depend on the anode surface roughness at given tube voltage and filtration.

6. I concluded that the surface roughness as a physical quantity alone is not adequate to describe the effect of surface roughness on the excited x-ray radiation. At small emission angles $(<\sim 6)$ and tube voltages $(<60 \mathrm{kVp})$ the ordering of spectra by anode surface roughness values and by the spectral photon flux values is not the same. This difference in the ordering of spectra by different surfaces is more significant in the mammographic tube voltage range $(25-40 \mathrm{kVp})$.

\section{Publications}

Publications related to the theses:

[P1] M. Erdélyi, M. Lajkó, R. Kákonyi, and G. Szabó, "Measurement of the x-ray tube anodes' surface profile and its effects on the x-ray spectra," Med. Phys. 36, 587-593 (2009).

[P2] R. Kákonyi, M. Erdélyi, and G. Szabó, "Monte Carlo analysis of energy dependent anisotropy of bremsstrahlung x-ray spectra,” Med. Phys. 36, 3897-905 (2009).

[P3] R. Kákonyi, M. Erdélyi, and G. Szabó, „Monte Carlo simulation of the effects of anode surface roughness on x-ray spectra," Med. Phys. 37, 5737-45 (2010). 